\title{
COMPLEXO: identifying the missing heritability of breast cancer via next generation collaboration
}

\author{
COMPLEXO: Melissa C Southey*, Daniel J Park', Tu Nguyen-Dumont', lan Campbell², Ella Thompson², Alison H Trainer, \\ Georgia Chenevix-Trench ${ }^{4}$, Jacques Simard ${ }^{5}$, Martine Dumont ${ }^{5}$, Penny Soucy ${ }^{5}$, Mads Thomassen ${ }^{6}$, Lars Jønson?

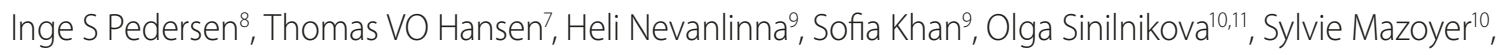 \\ Fabienne Lesueur' ${ }^{12}$, Francesca Damiola10, Rita Schmutzler ${ }^{13,14}$, Alfons Meindl15 ${ }^{15}$ Eric Hahnen ${ }^{13,14}$, Michael R Dufault ${ }^{15}$, \\ TL Chris Chan ${ }^{16,17}$, Ava Kwong ${ }^{16,18}$, Rosa Barkardóttir' ${ }^{19}$, Paolo Radice ${ }^{20}$, Paolo Peterlongo ${ }^{21}$, Peter Devilee ${ }^{22}$, \\ Florentine Hilbers ${ }^{22}$, Javier Benitez ${ }^{23}$, Anders Kvist24, Therese Törngren ${ }^{24}$, Douglas Easton ${ }^{25}$, David Hunter ${ }^{26}$, \\ Sara Lindstrom², Peter Kraft' ${ }^{26}$, Wei Zheng ${ }^{27}$, Yu-Tang Gao ${ }^{28}$, Jirong Long ${ }^{27}$, Susan Ramus ${ }^{29}$, Bing-Jian Feng ${ }^{30}$, \\ Jeffrey N Weitze ${ }^{31}$, Katherine Nathanson ${ }^{32}$, Kenneth Offit ${ }^{33}$, Vijai Joseph ${ }^{33}$, Mark Robson ${ }^{33}$, Kasmintan Schrader ${ }^{33}$, \\ San Ming Wang ${ }^{34}$, Yeong C Kim ${ }^{34}$, Henry Lynch ${ }^{35}$, Carrie Snyder ${ }^{35}$, Sean Tavtigian ${ }^{36}$, Susan Neuhausen ${ }^{37}$, \\ Fergus J Couch ${ }^{38}$ and David E Goldgar ${ }^{36}$
}

Linkage analysis, positional cloning, candidate gene mutation scanning and genome-wide association study approaches have all contributed significantly to our understanding of the underlying genetic architecture of breast cancer. Taken together, these approaches have identified genetic variation that explains approximately $30 \%$ of the overall familial risk of breast cancer, implying that more, and likely rarer, genetic susceptibility alleles remain to be discovered.

The application of massively parallel sequencing has further demonstrated the complexity of human genetic variation and has raised many challenges for computational and statistical methods for searching for additional breast cancer predisposition genes. Early findings are consistent with previous indications that no single gene is likely to account for a large proportion of the remaining unexplained genetic susceptibility $[1,2]$.

Coordinated international collaboration offers great potential to advance the discovery of additional breast cancer susceptibility genes by increasing the likelihood of identifying functionally relevant genetic variants in the same genes in multiple families. A new consortium, COMPLEXO (a name chosen to reflect the complexity of the exome), has been formed to facilitate collaborations between researchers actively applying massively parallel

*Correspondence: msouthey@unimelb.edu.au

'Genetic Epidemiology Laboratory, Department of Pathology, The University of

Melbourne, Victoria 3010, Australia

Full list of author information is available at the end of the article sequencing to understand the genetics of breast and ovarian cancer. The consortium has defined activities aimed at bringing together data and resources suitable for exome/genome sequencing initiatives and for large case-control-family study resources suitable for validation of candidate susceptibility genes in which rare mutations are associated with high to moderate risk of breast cancer. The aim of COMPLEXO is to bring to massively parallel sequencing the same power of large sample sets that have proven so successful in examining the role of common variants in cancer populations via the consortium model, such as the Breast Cancer Association Consortium (BCAC), the Consortium of Investigators of Modifiers of BRCA1/2 (CIMBA), the Ovarian Cancer Association Consortium (OCAC) and the Collaborative Oncology Gene-environment Study (COGS) [3-5]. However, sequencing studies provide additional challenges in terms of defining specific modes of collaboration given differences in sequencing and targeted capture platforms, bioinformatics platforms, the need to integrate ongoing studies in many centers and socio-ethical-legal issues that are not as relevant to initiatives that are genotyping common genetic variation.

COMPLEXO invites collaboration from any researcher who would like to contribute to this consortium either by contributing data to the combined COMPLEXO data set, contributing resources for large-scale validation of candidate breast cancer predisposition genes or refining analytical and bioinformatic pipelines for massively parallel sequencing data filtering and prioritization. COMPLEXO also has interests in the critical assessment of current platforms and protocols and in developing and improving 
data filtering and gene prioritization strategies to enhance gene discovery initiatives. These approaches are relevant to all complex human diseases.

Interested researchers can engage with COMPLEXO via any local member or by contacting the corresponding author.

\section{Competing interests}

The authors declare that they have no competing interests.

\section{Acknowledgements}

MCS is a National Health and Medical Research Council (Australia), Senior Research Fellow and Victorian Breast Cancer Research Consortium Group Leader. TN-D is a Susan G Komen for the Cure Postdoctoral Fellow. FJC is supported by the Breast Cancer Research Foundation. JB is the Head of Human Cancer Genetics Programme and coordinator of the Familial Cancer Exome Project in the Network of Research in Rare Diseases (CIBERER). SLN is the Morris and Horowitz Families Professor in Cancer Etiology and Outcomes Research. IGC is an NHMRC Principal Research Fellow. KO acknowledges grant support from Breast Cancer Reseach Foundation, Geoffrey Beene Cancer Research Foundation and STARR Cancer Consortium. JS is Chairholder of the Canada Research Chair in Oncogenetics. ERT is the recipient of a National Breast Cancer Foundation (Australia) Postdoctoral Training Fellowship. Support was received from R01CA155767 and the Victorian Breast Cancer Research Consortium.

\section{Author details}

'Genetic Epidemiology Laboratory, Department of Pathology, The University of Melbourne, Victoria 3010, Australia. ${ }^{2}$ Cancer Genetics Laboratory, Peter MacCallum Cancer Centre, East Melbourne, Victoria, Australia; Sir Peter MacCallum Department of Oncology and Department of Pathology, University of Melbourne, Parkville, Victoria, Australia. ${ }^{3}$ Familial Cancer Center, The Peter MacCallum Cancer Centre, St Andrew's Place, East Melbourne, Victoria 3010, Australia and Familial Cancer Centre, The Royal Melbourne Hospital, Parkville, Victoria, 3050, Australia. ${ }^{4}$ The Queensland Institute of Medical Research, Locked Bag 2000, Royal Brisbane Hospital, Herston, QLD 4029, Australia. ${ }^{5}$ Cancer Genomics Laboratory, Centre Hospitalier de Québec Research Center and Laval University, 2705 Laurier Boulevard, Quebec City, Quebec, Canada G1V 4G2. ${ }^{6}$ Department of Clinical Genetics, Odense University Hospital, Soenderboulevard 29, 5000 Odense C, Denmark. ${ }^{7}$ Center for Genomic Medicine, Rigshospitalet, University of Copenhagen, Copenhagen, Denmark. ${ }^{8}$ Department of Clinical Biochemistry, Section of Molecular Diagnostics, Aalborg University Hospital, Reberbansgade 15, 9000 Aalborg, Denmark. ${ }^{9}$ Department of Obstetrics and Gynecology, University of Helsinki and Helsinki University Central Hospital, Biomedicum Helsinki 4th floor PO BOX 700, 00029 HUS, Finland. ${ }^{10} \mathrm{CNRS}$ UMR5286 INSERM U1052, Universite' Lyon 1, Cancer Research Center of Lyon, Center Leon Berard, Lyon, France. ${ }^{11}$ Unite Mixte de Genetique Constitutionnelle des Cancers Frequents, Hospices Civils de Lyon, Centre Leon Berard, Lyon, France. ${ }^{12}$ INSERM, Unité U900, Mines ParisTech, Equipe Epidémiologie Génétique des Cancers, Institut Curie, 26 rue d'Ulm, 75248 Paris cedex 05, France. ${ }^{13} \mathrm{Center}$ of Familial Breast and Ovarian Cancer, University Hospital of Cologne, Cologne, Germany. ${ }^{14}$ Center for Molecular Medicine Cologne (CMMC), University of Cologne, Cologne, Germany. ${ }^{15}$ Department of Gynaecology and Obstetrics, Klinikum rechts der Isar at the Technical University, Munich, Germany. ${ }^{16}$ Hong Kong Hereditary Breast Cancer Family Registry, Hong Kong SAR. ${ }^{17}$ Department of Molecular Pathology, Hong Kong Sanatorium and Hospital, Hong Kong SAR and Departments of Pathology and Surgery, The University of Hong Kong, Hong Kong SAR. ${ }^{18}$ Department of Surgery, The University of Hong Kong, Hong Kong SAR; Department of Surgery, Hong Kong Sanatorium and Hospital, Hong Kong SAR; Department of Oncology, Stanford University School of Medicine, Stanford, California, United States of America. ${ }^{19}$ Department of Pathology, Landspitali-
University Hospital, Hringbraut, 101, Reykjavik, Iceland and BMC, Faculty of Medicine, University of Iceland, Vatnsmyrarvegi 16, 101, Reykjavik, Iceland. ${ }^{20}$ Unit of Molecular Bases of Genetic Risk and Genetic Testing, Department of Preventive and Predictive Medicine, Fondazione IRCCS Istituto Nazionale Tumori (INT), Milan, Italy. ${ }^{21}$ IFOM, Fondazione Istituto FIRC di Oncologia Molecolare, Milan, Italy. ${ }^{22}$ Department of Human Genetics, Leiden University Medical Center, Leiden, 2300 RC Leiden and Department of Pathology, Leiden University Medical Center, Leiden, 2300 RC Leiden, The Netherlands. ${ }^{23}$ Human Genetics Group, Human Cancer Genetics Programme, Spanish National Cancer Center (CNIO), E-28029 Madrid, Spain and Spanish Network on Rare Diseases (CIBERER), Valencia 46010, Spain. ${ }^{24}$ Department of Oncology, Clinical Sciences, Lund, University and Skåne University Hospital, Lund 22100, Sweden. ${ }^{25}$ Centre for Cancer Genetic Epidemiology, Department of Public Health and Primary Care and Department of Oncology, University of Cambridge, Strangeways Research Laboratory, Worts Causeway, Cambridge, CB1 8RN, UK. ${ }^{26}$ Harvard School of Public Health, 677 Huntington Avenue, Boston MA, 02115 , USA. ${ }^{27}$ Division of Epidemiology, Vanderbilt University School of Medicine, 2525 West End Avenue, Suite 800, Nashville, TN 37203, USA. ${ }^{28}$ Department of Epidemiology, Shanghai Cancer Institute, Shanghai, China. ${ }^{29}$ Department of Preventive Medicine, University of Southern California, Los Angeles, CA 90033, USA. ${ }^{30}$ Department of Dermatology, University of Utah School of Medicine, Salt Lake City, Utah, USA. ${ }^{31}$ Division of Clinical Cancer Genetics, City of Hope, 1500 E Duarte Rd, Duarte CA 91010, and the Clinical Cancer Genetics Community Research Network, USA. ${ }^{32}$ Translational Medicine and Human, Genetics and Abramson Cancer Center, Perelman School of Medicine at the University of Pennsylvania, Philadelphia, PA 19104, USA. ${ }^{33}$ Clinical Genetics Service, Department of Medicine, Memorial Sloan-Kettering Cancer Center, New York, NY 10065, USA. ${ }^{34}$ Department of Preventive Medicine, Creighton University School of Medicine, Omaha, NE 68178, USA. ${ }^{35}$ Department of Genetics, Cell Biology \& Anatomy, College of Medicine University of Nebraska Medical Center, 985145 Nebraska Medical Center, Omaha, NE 68198-5145, USA. ${ }^{36}$ Huntsman Cancer Institute, The University of Utah School of Medicine, Salt Lake City, UT 84112, USA. ${ }^{37}$ Department of Population Sciences, Beckman Research Institute of City of Hope, Duarte, CA 91010, USA. ${ }^{38}$ Division of Experimental Pathology, Department of Laboratory Medicine. and Pathology, Mayo Clinic, 200 First Street SW, Rochester, MN 55905, USA.

Published: 21 June 2013

\section{References}

1. Thompson ER, Doyle MA, Ryland GL, Rowley SM, Choong DY, Tothill RW Thorne H; kConFab, Barnes DR, Li J, Ellul J, Philip GK, Antill YC, James PA, Trainer AH, Mitchell G, Campbell IG: Exome sequencing identifies rare deleterious mutations in DNA repair genes FANCC and BLM as potential breast cancer susceptibility alleles. PLoS Genet 2012, 8:e1002894.

2. Park DJ, Lesueur F, Nguyen-Dumont T, Pertesi M, Odefrey F, Hammet F, Neuhausen SL, John EM, Andrulis IL, Terry MB, Daly M, Buys S, Le Calvez-Kelm F, Lonie A, Pope BJ, Tsimiklis H, Voegele C, Hilbers FM, Hoogerbrugge N, Barroso A, Osorio A; Breast Cancer Family Registry; Kathleen Cuningham Foundation Consortium for Research into Familial Breast Cancer, Giles GG, Devilee P, Benitez J, Hopper JL, Tavtigian SV, Goldgar DE, Southey MC: Rare mutations in XRCC2 increase the risk of breast cancer. Am J Hum Genet 2012, 90:734-739.

3. [http://ccge.medschl.cam.ac.uk/consortia/bcac/index.html]

4. CIMBA (The Consortium of Investigators of Modifiers of BRCA1/2) [http://ccge.medschl.cam.ac.uk/consortia/cimba/index.html]

5. Collaborative Oncological Gene-environment Study [http://www.cogseu.org]

doi:10.1186/bcr3434

Cite this article as: Southey MC, et al.: COMPLEXO: identifying the missing heritability of breast cancer via next generation collaboration. Breast Cancer Research 2013, 15:402. 